\title{
Generalized Serre Problem over Elementary Divisor Rings
}

\author{
Licui Zheng, ${ }^{1}$ Jinwang Liu, ${ }^{2}$ and Weijun Liu ${ }^{1,3}$ \\ ${ }^{1}$ School of Mathematics and Statistics, Central South University, Changsha 410075, China \\ ${ }^{2}$ Department of Mathematics and Computing Sciences, Hunan University of Science and Technology, Xiangtan, Hunan 411201, China \\ ${ }^{3}$ School of Science, Nantong University, Jiangshu 226019, China
}

Correspondence should be addressed to Weijun Liu; wjliu6210@126.com

Received 16 July 2015; Accepted 20 September 2015

Academic Editor: Laura Gardini

Copyright (C) 2015 Licui Zheng et al. This is an open access article distributed under the Creative Commons Attribution License, which permits unrestricted use, distribution, and reproduction in any medium, provided the original work is properly cited.

Matrix factorization has been widely investigated in the past years due to its fundamental importance in several areas of engineering. This paper investigates completion and zero prime factorization of matrices over elementary divisor rings (EDR). The Serre problem and Lin-Bose problems are generalized to EDR and are completely solved.

\section{Introduction}

In engineering and communication sciences, polynomial matrices are used in several different areas including circuits, multidimensional systems, controls, signal processing, and other areas. The Serre problem (or Serre Theorem) stands for a fundamental breakthrough in the understanding of polynomial matrices, and it is a powerful mathematical tool for engineers in practical designs. Following the work of Youla and Gnavi [1] on the basic structure of $n$-D system theory, many papers have been published in studying various prime factorization of multivariate polynomial matrices.

Lin and Bose in 2001 [2] formulated a generalized Serre conjecture for the polynomial ring $k\left[x_{1}, x_{2}, \ldots, x_{n}\right]$ over a field $k$. They found out that zero prime matrix completion and matrix primitive factorization were all related to the generalized Serre conjecture. So they proposed the existence problem of zero prime factorization for $n$-D polynomial matrices, which is now called Lin-Bose problem and it has been solved in [3-5].

We are interested in generalizing Serre conjecture and Lin-Bose problem to elementary divisor rings, which is defined in the next section. For example, let $\left(r_{1}, r_{2}, \ldots, r_{n}\right)$ be any row vector with entries in $R$, and let $d$ be any maximal common divisor of $r_{1}, \ldots, r_{n}$. We want to know if the row can be completed to a square matrix whose determinant is $d$. More generally, we will solve both Serre problem and
Lin-Bose problem for an arbitrary matrix (not just a row) over elementary divisor rings.

The organization of the paper is as follows. In Section 2, we first give some basic notions and describe Serre Problem and Lin-Bose problem precisely. In Section 3, we give proofs for the problems proposed in Section 2. Finally, a brief conclusion is given in Section 4 .

\section{Basic Notions and Main Problems}

Let $R$ be a commutative ring with a unity element 1 and $M_{\ell \times m}(R)$ the free module of $\ell \times m$ matrices with entries in $R$. For any $A \in M_{\ell \times m}(R), I_{t}(A)$ denotes the ideal of $R$ generated by all $t \times t$ minors of $A$, where $1 \leq t \leq \min (\ell, m)$. Set $I_{0}(A)=0$. The rank of $A$ is defined to be

$$
\operatorname{rank}(A)=\max \left\{t: 0 \leq t \leq r, \operatorname{Ann}_{R}\left(I_{t}(A)\right)=(0)\right\},
$$

where $\operatorname{Ann}_{R}\left(I_{t}(A)\right)=\left\{r \in R: r c=0\right.$ for all $\left.c \in I_{t}(A)\right\}$. When $\operatorname{rank}(A)=\ell$, we say $A$ is of full row rank.

Definition 1. A commutative ring $R$ is called an elementary divisor ring (EDR) if, for every $m \leq n$ and every matrix $A \epsilon$ $M_{m \times n}(R)$, there exist $P \in G L_{m}(R)$ and $Q \in G L_{n}(R)$ such that $P A Q=D$ with $D=\left(d_{i j}\right)$ is diagonal and every $d_{i i}$ divides $d_{i+1, i+1}$. 
When $m=1, A$ is row vector $\left(a_{1}, a_{2}, \ldots, a_{n}\right)$; the requirement $P A Q=D$ implies that the ideal $a_{1} R+a_{2} R+$ $\cdots+a_{n} R$ is generated by one element. So, in an elementary divisor ring, every finitely generated ideal is generated by one element. Note that an elementary divisor ring may not be a principle ideal domain, nor a unique factorization domain.

Definition 2. Let $F \in R^{\ell \times m}$ (or $F \in R^{m \times \ell}$ ), where $\ell \leq m$, be of full row rank. Then $F$ is said to be

(i) ZLP (or ZRP) if all $\ell \times \ell$ minors of $F$ generate the unit ideal $R$;

(ii) MLP if all $\ell \times \ell$ minors of $F$ are relatively prime; that is, $d(F)$ is a unit in $R$, where $d(F)$ refer to the maximal common divisor of all $\ell \times \ell$ minors of $F$.

Definition 3. Let $a_{1}, a_{2}, \ldots, a_{n} \in R$. $d$ is said to be a common divisor of $a_{1}, a_{2}, \ldots, a_{n}$ if $d \mid a_{i}, i=1,2, \ldots, n$. When $d$ is divisible by every common divisor of $a_{1}, a_{2}, \ldots, a_{n}$, one says that $d$ is a maximal common divisor of $a_{1}, a_{2}, \ldots, a_{n}$.

Note that, for any two maximal common divisors of $a_{1}, a_{2}, \ldots, a_{n}$, since they divide each other, they are always associates of each other; that is, they are different only by a factor that is invertible in $R$. Let $D\left(a_{1}, \ldots, a_{n}\right)$ denote the set of all maximal common divisors of $a_{1}, \ldots, a_{n}$.

Lemma 4. Let $R$ be an elementary divisor ring and $a_{1}, \ldots, a_{n} \in$ $R$. For any $d \in R$, one has $d \in D\left(a_{1}, \ldots, a_{n}\right)$ if and only if $a_{1} R+\cdots+a_{n} R=d R$.

Proof. First suppose $a_{1} R+\cdots+a_{n} R=d R$. Since $R$ has a unity element 1 , we have $a_{i}=a_{i} \cdot 1 \in a_{i} R \subset d R$, so $d \mid a_{i}$ for $i=1, \ldots, n$. Also, $a_{1} r_{1}+\cdots+a_{n} r_{n}=d$ for some $r_{i} \in R$; hence, for any $b \in R$ such that $b \mid a_{i}$ for $i=1, \ldots, n$, we must have $b \mid d$. Thus $d \in D\left(a_{1}, \ldots, a_{n}\right)$.

Next suppose $d \in D\left(a_{1}, \ldots, a_{n}\right)$. Then $a_{i}=d r_{i}$ for some $r_{i} \in R, i=1,2, \ldots, n$, so $a_{1} R+\cdots+a_{n} R \subseteq d R$. Since $R$ is an EDR, there exists $e \in R$ such that $a_{1} R+\cdots+a_{n} R=e R$. This implies that $e \mid a_{i}, i=1, \ldots, n$, so $e \mid d$; thus $d \in e R$ and $d R \subseteq e R=a_{1} R+\cdots+a_{n} R$. Therefore, $a_{1} R+\cdots+a_{n} R=d R$.

A direct consequence of the above lemma is that, in an elementary divisor ring, any collection of elements $a_{1}, a_{2}, \ldots, a_{n} \in R$ has at least one maximal common divisor, since the ideal $a_{1} R+\cdots+a_{n} R$ is generated by one element. This means that, for a unimodular row $\left(a_{1}, a_{2}, \ldots, a_{n}\right)$, the maximal common divisors of $a_{1}, a_{2}, \ldots, a_{n}$ must be units.

Definition 5. Let $F \in M_{\ell \times m}(R)$ with $\ell \leq m$, and let $a_{1}, a_{2}, \ldots, a_{\beta}$ denote all $\ell \times \ell$ minors of $F$, where $\beta=m ! /(m-$ $\ell) ! \ell$ !. Assume that there exists a maximal common divisor $d(F)$ of $a_{1}, a_{2}, \ldots, a_{\beta}$. Let $b_{i}$ be such that $a_{i}=d b_{i}, i=$ $1,2, \ldots, \beta$. Then $b_{1}, b_{2}, \ldots, b_{\beta}$ are called the "reduced minors" of $F$ with respect to $d$.

The original Serre problem and Lin-Bose problems are about the ring $A=k\left[x_{1}, x_{2}, \ldots, x_{n}\right]$, a polynomial ring in the variable $x_{1}, x_{2}, \ldots, x_{n}$ over a field $k$. More precisely, for any $F \in M_{\ell \times n}(A)(\ell \leq n)$ of full row rank, let $d(F)$ be the greatest common divisor of all $\ell \times \ell$ minors of $F$. Suppose all reduced minors of $F$ generate $A$. Then Serre's problem says that there exists a matrix $E \in A^{(m-\ell) \times \ell}$ such that $\operatorname{det}\left(\begin{array}{c}A \\ E\end{array}\right)=d(F)$. LinBose problem says that we can decompose $F$ as $F=D \cdot F_{1}$, where $D \in M_{\ell \times \ell}(A), F_{1} \in M_{\ell \times n}(A), \operatorname{det} D=d(F)$, and $F_{1}$ is ZLP.

In this paper, we extend the above two problems over to elementary divisor rings. Precisely, we completely solve the following problems.

Problem 6. Let $R$ be an elementary divisor ring and $A \in$ $M_{\ell \times n}(R)$, where $\ell \leq n$, is of full row rank. Let $d$ be a maximal common divisors of all $\ell \times \ell$ minors of $A$.

(a) (Serre) Is there a matrix $E \in A^{(m-\ell) \times \ell}$ such that $\operatorname{det}\left(\begin{array}{c}A \\ E\end{array}\right)=d$ ?

(b) (Lin-Bose) Is it possible to write $A$ as $A=F \cdot G_{1}$, where $F \in M_{\ell \times \ell}(R)$ with $\operatorname{det}(F)=d$ and $G_{1} \in M_{\ell \times n}(R)$ is ZLP?

\section{Main Results}

In this section, we give our main results. First, let us give some basic facts. For more details, we refer to [6]. Let $R$ be a commutative ring. Then any finite number of elements in $R$ have a maximal common divisor. Let $A \in M_{\ell \times m}(R)(\ell \leq m)$ be of full row rank. Let $a_{1}, a_{2}, \ldots, a_{t}$ 's be all of its $\ell \times \ell$ minors and $d$ the maximal common divisor of $a_{i}$ 's. Then there exists a matrix $H_{i} \in M_{m \times \ell}(R)$ such that $A H_{i}=a_{i} I_{\ell}=b_{i} d I_{\ell}$, where $a_{i}=b_{i} d$ and $I_{\ell}$ is the $\ell \times \ell$ identity matrix. Furthermore, if all the reduced minors of $A$ generate the unit ideal $R$, then there exists a matrix $H \in M_{m \times \ell}(R)$ such that $A \cdot H=d I_{\ell}$. When $\ell \geq m$, we have $H \cdot A=d I_{m}$

Lemma 7. Let $R$ be an EDR. Let $P \in M_{\ell \times n}(R)$ with $\ell \leq n$ with $d$ being a maximal common divisor of all $\ell \times \ell$ minors of $P$. Then, for every $F \in G L_{n}(R)$, the matrix $Q=P F$ also has $d$ as a maximal common divisor of its $\ell \times \ell$ minors.

Proof. Let $d^{\prime}$ be any maximal divisor of all $\ell \times \ell$ minors of $Q$ with $d^{\prime} \neq d$. Let $Q_{k}$ be any $\ell \times \ell$ submatrix of $Q$. Then $Q_{k}=P F_{k}$, where $F_{k}$ is a $n \times \ell$ submatrix of $F$. Then, by CauchyBinet formula, we can get that $\operatorname{det}\left(Q_{k}\right)=\sum \Delta_{i} \delta_{i}$, where $\Delta_{i}$ and $\delta_{i}$ are $\ell \times \ell$ minors of $P$ and $F_{k}$, respectively. Since, for every $i$, we have $d \mid \Delta_{i}$ and $d \mid \operatorname{det}\left(Q_{k}\right)$, by the arbitrariness of $Q_{k}$, we get $d \mid d^{\prime}$. Since $Q F^{-1}=P$, by the same reason, we have $d^{\prime} \mid d$, so $d R=d^{\prime} R$. Therefore, $d$ is also a maximal divisor of minors of $Q$.

Theorem 8. Let $R$ be an EDR and $A \in M_{\ell \times n}(R)$. Let $\left(r_{1}, r_{2}, \ldots, r_{n}\right)$ be an arbitrary row of $A$ and $d$ any maximal common divisor of $r_{1}, r_{2}, \ldots, r_{n}$. Then $\left(r_{1}, r_{2}, \ldots, r_{n}\right)$ can be completed to a square matrix

$$
\left(\begin{array}{c}
r_{1}, r_{2}, \ldots, r_{n} \\
C
\end{array}\right)
$$

whose determinant is $d$. Furthermore, the $(n-1) \times n$ matrix $C$ may be chosen to be itself completed to a matrix in $G L_{n}(R)$. 
Proof. Assume without loss of generality that $\left(r_{1}, r_{2}, \ldots, r_{n}\right)$ is the first row of $A$; then according to the definition of an elementary divisor ring, there exist $P_{1} \in G L_{\ell}(R)$ and $Q_{1} \in$ $G L_{n}(R)$ such that

$$
P_{1} A Q_{1}=\operatorname{diag}\left\{d_{1}, d_{2}, \ldots, d_{n}\right\} .
$$

By Lemma $4 d_{1}$ is a maximal common divisor of $\left(r_{1}, r_{2}, \ldots\right.$, $\left.r_{n}\right)$. Assume that $\operatorname{det}\left(P_{1}\right)=u$ and $\operatorname{det}\left(Q_{1}\right)=v$ are units in $R$. Let

$$
\begin{gathered}
P=\operatorname{diag}\left\{1, u^{-1}, \ldots, 1\right\} P_{1}, \\
Q=Q_{1} \cdot \operatorname{diag}\left\{1, v^{-1}, \ldots, 1\right\} .
\end{gathered}
$$

Then, $\operatorname{det}(P)=\operatorname{det}(Q)=1, P \in S L_{\ell}(R), Q \in S L_{n}(R)$, and

$$
P\left(\begin{array}{c}
r_{1}, r_{2}, \ldots, r_{n} \\
A^{\prime}
\end{array}\right) Q=\operatorname{diag}\left\{d_{1}, d_{2}, \ldots, d_{n}\right\},
$$

where $A^{\prime}$ is the submatrix of $A$ formed by the remaining rows after removing $\left(r_{1}, r_{2}, \ldots, r_{n}\right)$ from $A$.

$$
\begin{aligned}
& \text { Set } C=P^{-1}\left(O_{(n-1) \times 1} I_{n-1}\right) Q^{-1} \text {. Then } \\
& P\left(\begin{array}{c}
r_{1}, r_{2}, \ldots, r_{n} \\
C
\end{array}\right) Q=\left(\begin{array}{cc}
d_{1} & 0_{1 \times(n-1)} \\
O_{(n-1) \times 1} & I_{n-1}
\end{array}\right)
\end{aligned}
$$

Note that $\operatorname{det}(P)=\operatorname{det}(Q)=1$. Thus

$$
\operatorname{det}\left(\begin{array}{c}
r_{1}, r_{2}, \ldots, r_{n} \\
C
\end{array}\right)=\operatorname{det}\left(\begin{array}{cc}
d_{1} & 0_{1 \times(n-1)} \\
O_{(n-1) \times 1} & I_{n-1}
\end{array}\right)=d_{1} .
$$

So $C$ is ZLP and can be completed to matrix in $G L_{n}(R)$.

By Lemma 4, $d R=a_{1} R+a_{2} R+\cdots+a_{n} R=d_{1} R$; we have that $d=d_{1} x$ and $d_{1}=d y$, where $x, y \in R$. Then

$$
(d, 0)\left(\begin{array}{cc}
y & 1-y x \\
-1 & x
\end{array}\right)=\left(d_{1}, 0\right) \text {. }
$$

This proves the theorem.

In the above theorem, $d$ is an arbitrary maximal common divisor, but $R$ is not UFD so the maximal common divisors are not unique. If $d$ is a beforehand given maximal common divisor, is the above theorem also correct? The following theorem gives a positive answer.

Theorem 9. Let $R$ be an EDR and $F \in M_{\ell \times n}(R)$ with $\ell \leq n$. Then there exist $A \in M_{\ell \times \ell}(R)$ and $F_{1} \in M_{\ell \times n}(R)$ such that $F=A \cdot F_{1}$, where $\operatorname{det}(A)$ is a maximal common divisor of all $\ell \times \ell$ minors of $F$ and $F_{1}$ is $Z L P$.

Proof. Since $R$ is an elementary divisor ring, there exist $P \in$ $S L_{l}(R)$ and $Q \in S L_{n}(R)$ such that

$$
P F Q=\left(D_{1}, 0\right), \quad \text { where } D_{1}=\operatorname{diag}\left\{d_{1}, d_{2}, \ldots, d_{\ell}\right\},
$$

and every $d_{i}$ is a divisor of $d_{i+1}$, and $0 \in M_{\ell \times(n-\ell)}(R)$. By Lemma $7, \operatorname{det}\left(D_{1}\right)=d_{1} d_{2} \cdots d_{\ell}$ is a maximal common divisor of all $\ell \times \ell$ minors of $A$. Partition $Q^{-1}$ as

$$
\left(\begin{array}{l}
Q_{1} \\
Q_{2}
\end{array}\right) \text {, where } Q_{1} \in M_{l \times n}(R), Q_{2} \in M_{(n-\ell) \times n}(R) \text {. }
$$

Let $A=P^{-1} D_{1}$. Since $Q^{-1} \in S L_{n}(R)$, by Laplace expansion, $Q_{1}$ is ZLP. Since $F=P^{-1}\left(D_{1}, 0\right) Q^{-1}$, we have $\operatorname{det}(A)=$ $\operatorname{det}\left(P^{-1} D_{1}\right)=d_{1} d_{2} \cdots d_{\ell}$. Hence $F=A \cdot Q_{1}$, as claimed by the theorem.

Theorem 10. With the same notation as in the proof of the above theorem, the maximal divisor of all $i \times i$ minors of $A$ is $d_{1} \cdots d_{i}$ for $1 \leq i \leq \ell$.

Proof. First, we take care of the $1 \times 1$ minors of $A$, which is $d_{1} a_{i j}$ as $A=P^{-1} D_{1}$, where $P^{-1}=\left(a_{i j}\right)$. Assume that $u$ is a common divisor of $a_{i j}, u a_{i j}^{\prime}=a_{i j}$. Then

$$
\operatorname{det}\left(P^{-1}\right)=\sum_{s=1}^{n} a_{i s} A_{i s}=u \sum_{s=1}^{n} a_{i s}^{\prime} A_{i s}^{\prime}=1,
$$

where $A_{i s}$ is $(\ell-1) \times(\ell-1)$ minors of $P^{-1}$ and $u A_{i s}^{\prime}=A_{i s}$. Then we can get that $u$ is a unit of $R$. It follows that the maximal divisor of all $1 \times 1$ minors of $A$ is $d_{1}$.

Now let $i \geq 2$. Suppose that the result is correct for $(i-1) \times$ $(i-1)$ minors. We investigate this result for $i \times i$ minors. Let $C_{1}, \ldots, C_{k}$ be all the $i \times i$ minors and $B_{1}, \ldots, B_{m}$ all the $(i-1) \times$ $(i-1)$ submatrix of $C_{1}, \ldots, C_{k}$, where $m=n ! /(n-i+1) !(i-1)$ ! and $k=n ! /(n-i) !(i) !$. Then, by the Laplace expansion,

$$
\operatorname{det}\left(C_{j}\right)=\sum_{t=1}^{i} d_{i} a_{t, i} B_{j t}
$$

But the common divisor of $a_{i j}$ is a unit of $R$, and the common divisor of $B_{1}, \ldots, B_{m}$ is $d_{1} \cdots d_{i-1}$, so the common divisor of $C_{1}, \ldots, C_{k}$ is $d_{1} \cdots d_{i}$. The theorem follows by induction on $i$.

Besides, we can also make some improvements for the above theorem, which can be seen as the Serre problem generalized to elementary divisor rings.

Theorem 11. Let $R$ be an EDR and $F \in M_{\ell \times n}(R)(\ell \leq n)$ of full row rank. Then, for any maximal common divisor $d$ of all $\ell \times \ell$ minors of $F$, there exist $A \in M_{\ell \times \ell}(R)$ and $F_{1} \in M_{\ell \times n}(R)$ such that $F=A \cdot F_{1}$ with $\operatorname{det}(A)=d$ and $F_{1}$ is $Z L P$.

Proof. Assume $d$ and $d^{\prime}$ are two maximal common divisors of all $\ell \times \ell$ minors of $F$. By Lemma $4, d R=d^{\prime} R$, which means there exist $x, y \in R$ such that $d=d^{\prime} x$ and $d^{\prime}=d y$. Note that $I_{\ell}(F)=0$ as $F$ is of full row rank. Then $d$ and $d^{\prime}$ are not zerodivisors. Also, any two maximal common divisors of all $\ell \times \ell$ minors of $F$ are associates of each other; that is, there exists a unit $\alpha$ in $R$ such that $d=\alpha \cdot d^{\prime}$.

By Theorem 9, there exist $D_{2} \in M_{\ell \times \ell}(R)$ and $A_{2} \in$ $M_{l \times n}(R)$ such that $F=D_{2} \cdot A_{2}$, where $d_{1}=\operatorname{det}\left(A_{2}\right)$ is a maximal common divisor of all $\ell \times \ell$ minors of $A$, and $D_{2}$ is ZLP. Set $A=D_{2} \cdot \operatorname{diag}\{\alpha, 1, \ldots, 1\}$. Then $F_{1}=$ $\operatorname{diag}\left\{\alpha^{-1}, 1, \ldots, 1\right\} \cdot A_{2}$. Thus the theorem is proved.

Remark 12. The above two theorems are different from each other, as in Theorem $11 d$ is beforehand given, but in Theorem $9 d$ is an arbitrary one. 
By now, we proved that Lin-Bose problem over an elementary divisor ring is correct. Next we deal with the Serre problem.

Theorem 13. Let $R$ be an elementary divisor ring and $F \in$ $M_{\ell \times n}(R)$ with $\ell<n$. Then $F$ can be completed to a square matrix $\left(\begin{array}{c}F \\ G\end{array}\right)$ whose determinant is a maximal common divisor of all $\ell \times \ell$ minors of $F$. Furthermore, the $(n-\ell) \times n$ matrix $G$ may be completed to a matrix in $G L_{n}(R)$.

Proof. From Theorem 8, we have that $P F Q=(D, 0)$, where $P \in S L_{l}(R), Q \in S L_{n}(R), D=\operatorname{diag}\left\{d_{1}, d_{2}, \ldots, d_{\ell}\right\}$, and $\operatorname{det}(D)=d_{1} d_{2} \cdots d_{\ell}$. Set $G=P^{-1}\left(O_{(n-1) \times 1} I_{n-1}\right) Q^{-1}$. Then we have

$$
P\left(\begin{array}{l}
F \\
G
\end{array}\right) Q=\left(\begin{array}{cc}
D & 0_{1 \times(n-1)} \\
O_{(n-1) \times 1} & I_{n-1}
\end{array}\right) .
$$

This implies that

$$
\operatorname{det}\left(\begin{array}{l}
F \\
G
\end{array}\right)=\operatorname{det}\left(\begin{array}{cc}
D & 0 \\
0 & I_{n-\ell}
\end{array}\right)=\operatorname{det}(D)
$$

Furthermore, 1 is a maximal common divisor of all $(n-\ell) \times$ $(n-\ell)$ minors of $G$. From above argument, the $(n-\ell) \times n$ matrix $G$ may be chosen to be itself completable to a matrix in $G L_{n}(R)\left(S L_{n}(R)\right)$.

In this theorem $d$ is a particular maximal common divisor. When $d$ is an arbitrary maximal common divisor, this theorem is also correct.

Theorem 14. Let $R$ be an EDR, let $F \in M_{\ell \times n}(R)$ be of full row rank, and let $d$ be any maximal divisor of all $\ell \times \ell$ minors of $F$. Then $F$ can be completed to a square matrix $\left(\begin{array}{c}F \\ G\end{array}\right)$ whose determinant is $d$. Furthermore, the $(n-l) \times n$ matrix $G$ may be chosen to be itself completable to matrix in $G L_{n}(R)$.

Proof. From Lemma 4, any two maximal common divisors of all minors of $F$ are associates. From Theorem 8, there exist $P \in S L_{l}(R)$ and $Q \in S L_{n}(R)$ such that $P F Q=(D, 0)$, where $D=\operatorname{diag}\left\{d_{1}, d_{2}, \ldots, d_{\ell}\right\}$; every $d_{i}$ is a divisor of $d_{i+1}$, and $0 \in M_{\ell \times(n-\ell)}(R)$. By Lemma 7, $\operatorname{det}(D)$ is a maximal common divisor of all $\ell \times \ell$ minors of $F$. Assume $d=\varepsilon \cdot \operatorname{det}(D) ; \varepsilon$ is a unit in $R$. Set $\bar{Q}=Q \cdot \operatorname{diag}\{\varepsilon, 1, \ldots, 1\}$. Then $P F \bar{Q}=$ $(\bar{D}, 0)$, where $\bar{Q} \in G L_{n}(R)$ and $\operatorname{det}(\bar{D})=d$. Setting $G=$ $P^{-1}\left(0_{(n-\ell) \times \ell} I_{n-\ell}\right) \bar{Q}^{-1}$, we obtain the result.

Theorem 15. Let $R$ be an EDR and let $A, B \in M_{n \times n}(R)$ be of full row rank. Assume $\operatorname{det}(A B) \neq 0$, and

$$
\begin{gathered}
P_{1} A Q_{1}=D_{1}=\operatorname{diag}\left\{d_{1}, \ldots, d_{n}\right\}, \\
P_{2} B Q_{2}=D_{2}=\operatorname{diag}\left\{e_{1}, \ldots, e_{n}\right\}, \\
P_{3} A B Q_{3}=D_{3}=\operatorname{diag}\left\{f_{1}, \ldots, f_{n}\right\},
\end{gathered}
$$

where $P_{j}, Q_{j} \in G L_{n}(R)$ for $j=1,2,3$. Then $d_{i} \mid f_{i}$ and $e_{i} \mid f_{i}$ for all $1 \leq i \leq n$.
Proof. By Theorem 11, there exist $D \in M_{n \times n}(R)$ and $A_{1} \in$ $S L_{n}(R)$ such that $A=D A_{1}$. Then $\operatorname{det}(A)=\operatorname{det}(D)=$ $d_{1} \cdots d_{n}$. For $B$, we also have $B=E B_{1}$, and $\operatorname{det}(B)=\operatorname{det}(E)=$ $e_{1} \cdots e_{n}$, where $E \in M_{n \times n}(R)$ and $B_{1} \in S L_{n}(R)$. It follows that $A B=D A_{1} E B_{1}$ and $\operatorname{det}(A B)=\operatorname{det}(D E)=d_{1} \cdots d_{n} e_{1} \cdots e_{n}$.

Now for $A B$, there exist $F \in M_{n \times n}(R)$ and $H \in S L_{n}(R)$ such that $A B=F H$. Then $\operatorname{det}(A B)=\operatorname{det}(F)=f_{1} \cdots f_{n}$. So $d_{1} \cdots d_{n} e_{1} \cdots e_{n}=f_{1} \cdots f_{n}$. So $d_{i}$ and $e_{i}$ all divide $f_{1} \cdots f_{n}$ for $i=1, \ldots, n$.

We prove the theorem by induction on $n$. If $n=1$, it is obvious. Let $i \geq 1$. Suppose that the result is correct for $n=i$; we investigate this result for $n=i+1$. By the definition of EDR and the assumption, we may set

$$
\begin{gathered}
d_{2}=d_{1} x_{1}, \\
d_{3}=d_{1} x_{1} x_{2},
\end{gathered}
$$

$$
\begin{gathered}
d_{\ell}=d_{1} x_{1} x_{2} \cdots x_{\ell-1}, \\
d_{l+1}=d_{1} x_{1} x_{2} \cdots x_{\ell}, \\
f_{1}=d_{1} y_{1}, \\
f_{2}=d_{1} y_{1} y_{2}, \\
\vdots \\
f_{\ell}=d_{1} y_{1} y_{2} \cdots y_{\ell}, \\
f_{\ell+1}=d_{1} y_{1} y_{2} \cdots y_{\ell+1},
\end{gathered}
$$

where $x_{i}, y_{i} \in R$. By the above, $d_{1} d_{2} \cdots d_{\ell+1} \mid f_{1} f_{2} \cdots f_{\ell+1}$; it follows that

$$
\begin{gathered}
\left(d_{1} \cdot d_{1} x_{1} \cdot d_{1} x_{1} x_{2} \cdots d_{\ell} x_{1} x_{2} \cdots x_{\ell}\right) \mid \\
\left(d_{1} y_{1} \cdot d_{1} y_{1} y_{2} \cdots d_{\ell} y_{1} y_{2} \cdots y_{\ell+1}\right)
\end{gathered}
$$

Hence

$$
\left(x_{1} \cdot x_{1} x_{2} \cdot x_{1} x_{2} \cdots x_{\ell}\right) \mid\left(y_{1} \cdot y_{1} y_{2} \cdots y_{1} y_{2} \cdots y_{\ell+1}\right) \text {. }
$$

Since $d_{2}\left|f_{2}, d_{3}\right| f_{3}, \ldots, d_{\ell} \mid f_{\ell}$, we conclude that

$$
\begin{gathered}
x_{1} \mid y_{1} y_{2}, \\
x_{1} x_{2} \mid y_{1} y_{2} y_{3}, \\
\vdots \\
x_{1} x_{2} \cdots x_{l-1} \mid y_{1} y_{2} \cdots y_{l} .
\end{gathered}
$$

So there exist $q_{1}, q_{2}, \ldots, q_{\ell-1}$ such that

$$
\begin{gathered}
x_{1} q_{1}=y_{1} y_{2}, \\
x_{1} x_{2} q_{2}=y_{1} y_{2} y_{3}, \\
\vdots
\end{gathered}
$$$$
x_{1} x_{2} \cdots x_{\ell-1} q_{\ell-1}=y_{1} y_{2} \cdots y_{\ell} .
$$ 
That is,

$$
\begin{aligned}
& x_{1} \cdot x_{1} x_{2} \cdot x_{1} x_{2} \cdots x_{\ell} \mid y_{1} \cdot x_{1} q_{1} \cdots x_{1} x_{2} \cdots x_{\ell-1} q_{\ell-1} \\
& \cdot y_{1} y_{2} \cdots y_{\ell+1} .
\end{aligned}
$$

Thus

$$
x_{1} x_{2} \cdots x_{l} \mid y_{1} \cdot q_{1} q_{2} \cdots q_{\ell-1} \cdot y_{1} y_{2} \cdots y_{\ell+1} .
$$

Now, assume that $d_{\ell+1} \dagger f_{\ell+1}$. Then $x_{1} x_{2} \cdots x_{\ell} \dagger y_{1} y_{2} \cdots$ $y_{\ell+1}$, and $x_{1} x_{2} \cdots x_{\ell} \mid y_{1} \cdot q_{1} q_{2} \cdots q_{\ell-1}$. But

$$
\begin{gathered}
y_{1} \mid y_{1} y_{2} \cdots y_{\ell+1}, \\
q_{1} \mid y_{1} y_{2} \cdots y_{\ell+1}, \\
\vdots \\
q_{\ell-1} \mid y_{1} y_{2} \cdots y_{\ell+1} .
\end{gathered}
$$

Therefore, we have $x_{1} x_{2} \cdots x_{\ell} \mid y_{1} y_{2} \cdots y_{\ell+1}$, contradicting our assumption. So $d_{\ell+1} \mid f_{\ell+1}$, and our theorem is proved.

Theorem 16. Let $R$ be an EDR and let $F_{1}, F_{2} \in M_{\ell \times m}(R)(\ell \leq$ $m$ ) be offull row rank. Suppose $F_{1}=F_{2} U$, where $U \in M_{m \times m}(R)$ is MLP. Then $F_{1}$ is MLP if and only if $F_{2}$ is MLP.

Proof. Let $d_{1}$ and $d_{2}$ be maximal common divisors of all $\ell \times \ell$ minors of $F_{1}$ and $F_{2}$, respectively. Let $F_{k}$ be any $\ell \times \ell$ submatrix of $F_{1}$, and $F_{k}=F_{2} U_{k}$, where $U_{k}$ is a $m \times \ell$ submatrix of $U$. By Cauchy-Binet formula, we have $\operatorname{det}\left(F_{k}\right)=\sum a_{2 i} \beta_{i}$, where the sum is all $a_{2 i}$ and $\beta_{i}$, which are $\ell \times \ell$ minors of $F_{2}$ and $U_{k}$, respectively. Since $d_{2} \mid a_{2 i}$ for every $i$, we have $d_{2} \mid \operatorname{det}\left(F_{k}\right)$. Because $\operatorname{det}\left(F_{k}\right)$ is an arbitrary $\ell \times \ell$ minor of $F_{1}$, we have $d_{2} \mid$ $d_{1}$. As $U$ is MLP, from Lemma 7, there exist $V_{k} \in M_{m \times r}(R)$ such that $U V_{k}=\delta_{k} I_{r}$, where $\delta_{k}$ are $\ell \times \ell$ minors of $U$. Then, from $F_{1}=F_{2} U$, we get that $\delta_{k} F_{2}=F_{1} V_{k}$. By Cauchy-Binet formula, $d_{1} \mid \delta_{k}^{l} d_{2}$ for every $k$. $\operatorname{But} \operatorname{gcd}\left(\delta_{1}^{l}, \delta_{2}^{l}, \ldots, \delta_{r}^{l}\right)=1$ as $U$ is MLP, so $d_{1} \mid d_{2}$.

Therefore, if $F_{1}$ is MLP, then $d_{1}$ is a unit and so is $d_{2}$, which means that $F_{2}$ is also MLP. By similar reasoning, when $F_{2}$ is MLP, so is $F_{1}$.

Theorem 17. Let $R$ be an EDR and $F \in M_{m \times m+1}(R)$ is of full row rank. If $F$ is $Z L P$, then there exists a $Z R P$ matrix $b_{0} \in M_{m \times m-1}(R)$ such that $F b_{0}=0$.

Proof. Let $F=\left[\begin{array}{ll}U & v\end{array}\right]$, and set $P=U^{-1} v \in M_{m \times 1}(R)$. There exist $P_{1} \in G L_{l}(R)$ and $Q_{1} \in R$ such that $P_{1} P Q_{1}=$ $\operatorname{diag}\left(d_{1}, 0, \ldots, 0\right)$ as $R$ is an $E D R$, where $d_{1} \in R$. Then

$$
P=P_{1}^{-1} \operatorname{diag}\left(d_{1}, 0, \ldots, 0\right) Q_{1}^{-1} .
$$

Set $B=P_{1}^{-1} \operatorname{diag}\left(d_{1}, 0, \ldots, 0\right)$ and $a=Q_{1}$. Then $P=B a^{-1}$; that is, $B a^{-1}=U^{-1} v$, and $v a=U\left(U^{-1} v a\right)=U\left(B a^{-1}\right) a=U B$. It follows that

$$
\left[\begin{array}{ll}
U & v
\end{array}\right]\left[\begin{array}{c}
-B \\
a
\end{array}\right]=0 .
$$

Let $b_{0}=\left[\begin{array}{c}-B \\ a\end{array}\right]$. Then $b_{0} \in M_{m \times m-1}(R)$ and $F b_{0}=0$. Let $b_{1}, b_{2}, \ldots, b_{m}$ be the $(m-1) \times(m-1)$ minors of $F$. Then there exist $k \in R \backslash\{0\}$ such that $b_{i}=k c_{i}(1 \leq i \leq m)$ as $B a^{-1}=U^{-1} v$. Hence, if $F$ is ZLP, then $b_{0}$ is ZRP.

\section{Conclusions}

The main results in this paper can be summarized as follows: (a) the Serre problem and Lin-Bose problems were solved over an elementary divisor ring; (b) by using the properties of EDR, some interesting results about ZLP matrices are proved. These results could provide engineers with useful information for finding desired matrix decomposition.

\section{Conflict of Interests}

The authors declare that there is no conflict of interests regarding the publication of this paper.

\section{Acknowledgments}

This research is supported by the National Science Foundation of China (11471108, 11426101, and 11271208), Hunan Provincial Natural Science Foundation of China (14JJ6027 and 2015JJ2051), Scientific Research Fund of Hunan Province Education Department (15B082), and Fundamental Research Funds for the Central Universities of Central South University under Grant no. 2013zzts008.

\section{References}

[1] D. C. Youla and G. Gnavi, "Notes on n-dimensional system theory," IEEE Transactions on Circuits and Systems, vol. 26, no. 2, pp. 105-111, 1979.

[2] Z. Lin and N. K. Bose, "A generalization of Serre's conjecture and some related issues," Linear Algebra and Its Applications, vol. 338, no. 1-3, pp. 125-138, 2001.

[3] J. F. Pommaret, "Solving bose conjecture on linear multidimensional systems," in Proceedings of the European Control Conference (ECC '01), pp. 1853-1855, IEEE, Porto, Portugal, September 2001.

[4] M. Wang and D. Feng, "On Lin-Bose problem," Linear Algebra and Its Applications, vol. 390, no. 1-3, pp. 279-285, 2004.

[5] J. Liu, D. Li, and L. Zheng, "The lin-bose problem," IEEE Transactions on Circuits and Systems II: Express Briefs, vol. 61, no. 1, pp. 41-43, 2014.

[6] J. Liu and M. Wang, "New results on multivariate polynomial matrix factorizations," Linear Algebra and Its Applications, vol. 438, no. 1, pp. 87-95, 2013. 


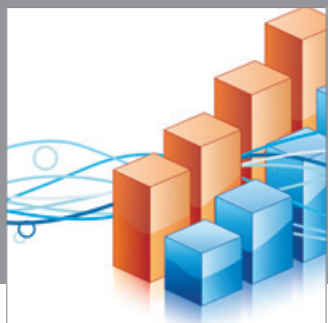

Advances in

Operations Research

mansans

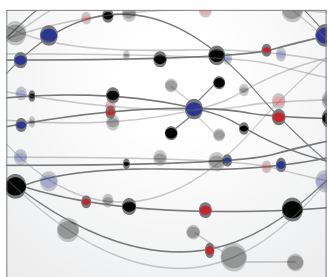

The Scientific World Journal
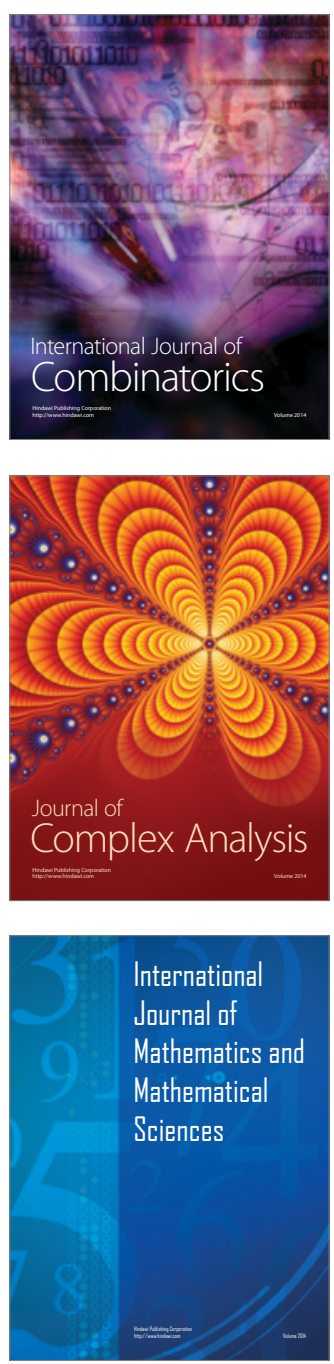
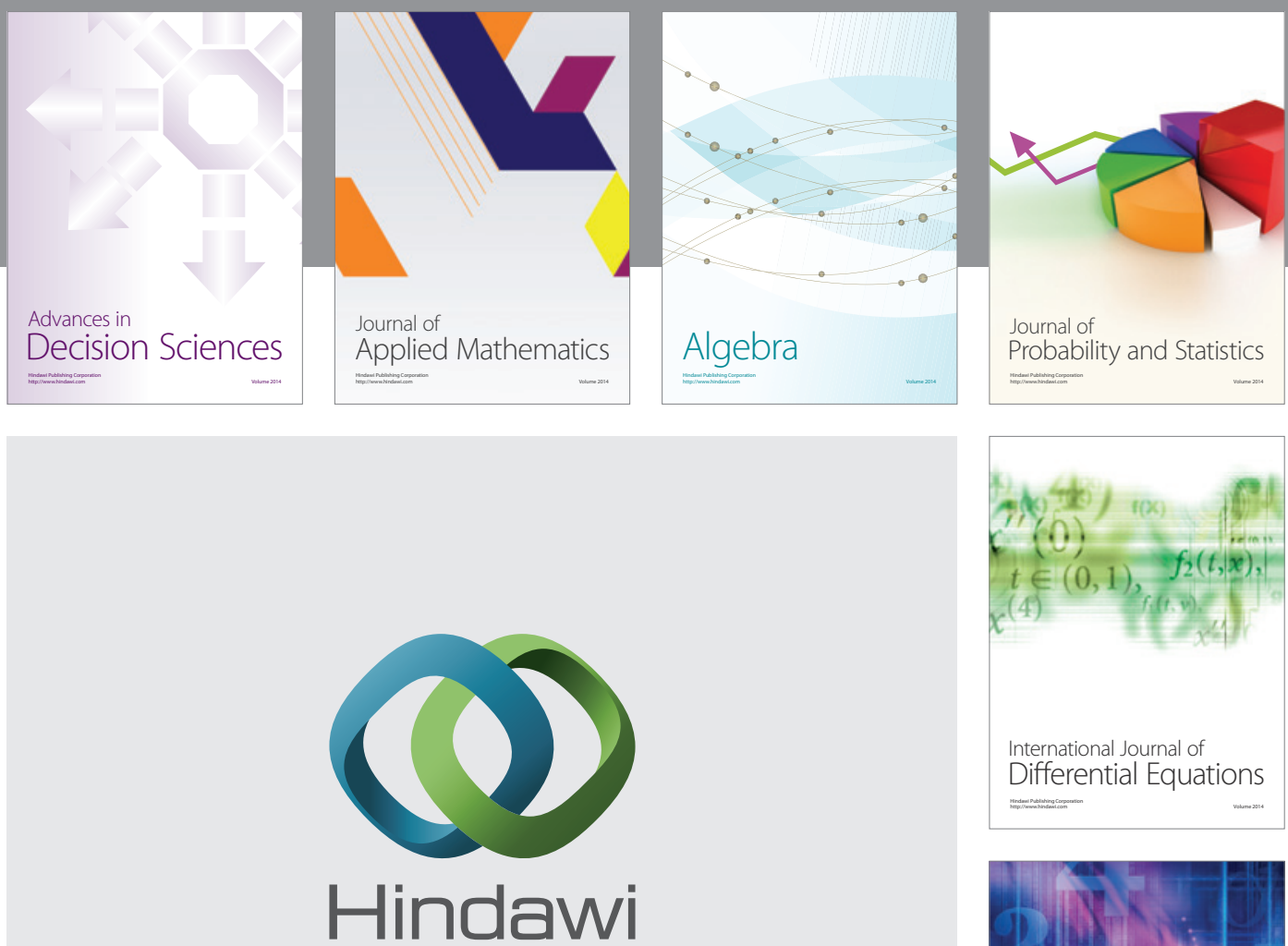

Submit your manuscripts at http://www.hindawi.com
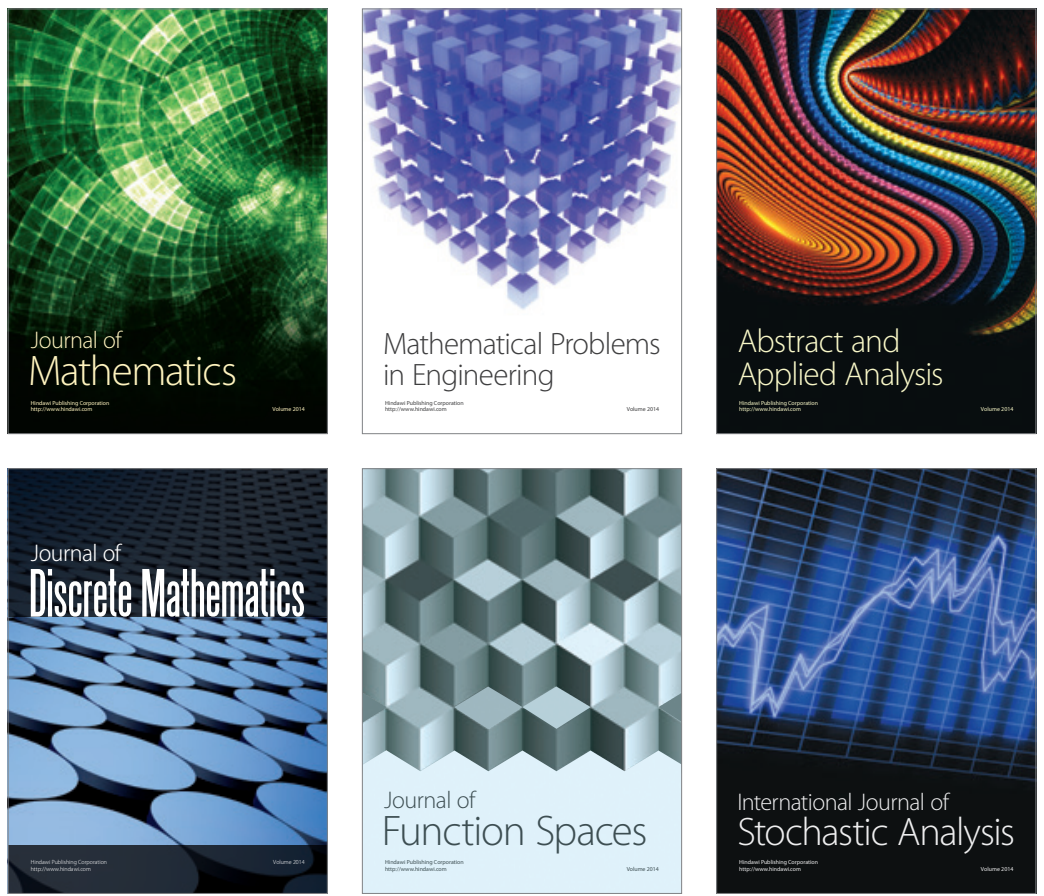

Journal of

Function Spaces

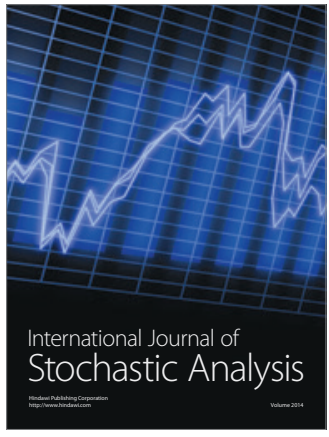

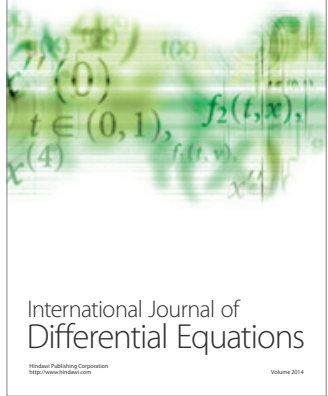
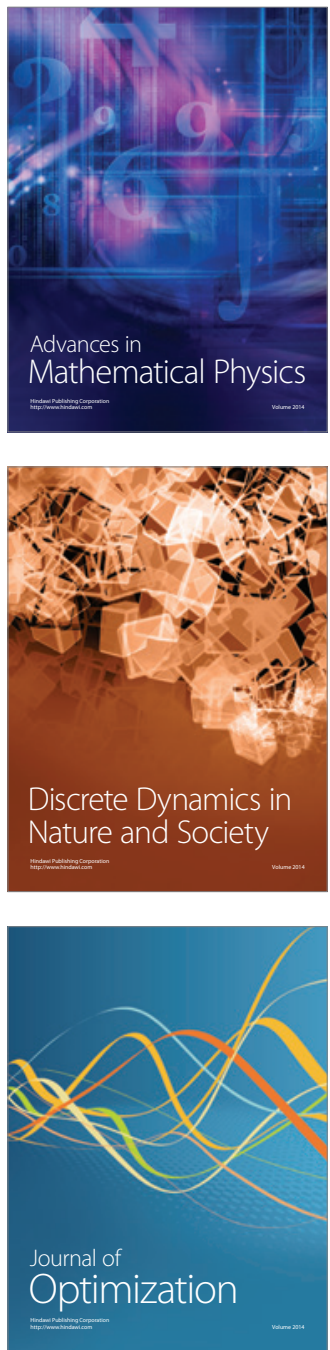